\title{
Solar radius determined from PICARD/SODISM observations and extremely weak wavelength dependence in the visible and the near-infrared
}

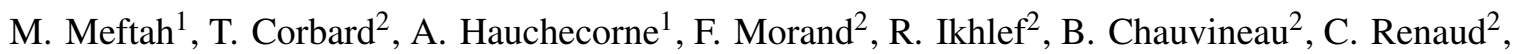 \\ A. Sarkissian ${ }^{1}$, and L. Damé ${ }^{1}$ \\ ${ }^{1}$ Université Paris Saclay, Sorbonne Université, UVSQ, CNRS, LATMOS, 11 Boulevard d'Alembert, 78280 Guyancourt, France \\ e-mail: Mustapha.Meftah@latmos.ipsl.fr \\ ${ }^{2}$ Université de la Côte d'Azur, Observatoire de la Côte d'Azur (OCA), CNRS, Boulevard de l'Observatoire, 06304 Nice, France
}

Received 23 October 2017 / Accepted 9 May 2018

\begin{abstract}
Context. In 2015, the International Astronomical Union (IAU) passed Resolution B3, which defined a set of nominal conversion constants for stellar and planetary astronomy. Resolution B3 defined a new value of the nominal solar radius $\left(R_{\odot}^{N}=695700 \mathrm{~km}\right)$ that is different from the canonical value used until now $(695990 \mathrm{~km})$. The nominal solar radius is consistent with helioseismic estimates. Recent results obtained from ground-based instruments, balloon flights, or space-based instruments highlight solar radius values that are significantly different. These results are related to the direct measurements of the photospheric solar radius, which are mainly based on the inflection point position methods. The discrepancy between the seismic radius and the photospheric solar radius can be explained by the difference between the height at disk center and the inflection point of the intensity profile on the solar limb. At $535.7 \mathrm{~nm}$ (photosphere), there may be a difference of $\sim 330 \mathrm{~km}$ between the two definitions of the solar radius.

Aims. The main objective of this work is to present new results of the solar radius in the near-ultraviolet, the visible, and the nearinfrared from PICARD space-based and ground-based observations. Simulations show the strong influence of atmosphere effects (refraction and turbulence) on ground-based solar radius determinations and highlight the interest of space-based solar radius determinations, particularly during planet transits (Venus or Mercury), in order to obtain more realistic and accurate measurements.

Methods. Solar radius observations during the 2012 Venus transit have been made with the SOlar Diameter Imager and Surface Mapper (SODISM) telescope on board the PICARD spacecraft. We used the transit of Venus as an absolute calibration to determine the solar radius accurately at several wavelengths. Our results are based on the determination of the inflection point position of the solar limb-darkening function (the most common solar radius definition). A realistic uncertainty budget is provided for each solar radius obtained with the PICARD space-based telescope during the 2012 Venus transit. The uncertainty budget considers several sources of error (detection of the centers of Venus and Sun in PICARD images, positions of Sun and Venus from ephemeris (planetary theory), PICARD on-board timing, PICARD spacecraft position, and optical distortion correction from PICARD images).

Results. We obtain new values of the solar radius from the PICARD mission at several wavelengths and in different solar atmosphere regions. The PICARD spacecraft with its SODISM telescope was used to measure the radius of the Sun during the Venus transit in 2012. At $535.7 \mathrm{~nm}$, the solar radius is equal to $696134 \pm 261 \mathrm{~km}$ (combined standard uncertainty based $(\xi)$ on the uncertainty budget). At $607.1 \mathrm{~nm}$, the solar radius is equal to $696156 \pm 145 \mathrm{~km}(\xi)$, and the standard deviation of the solar radius mean value is $\pm 22 \mathrm{~km}$. At $782.2 \mathrm{~nm}$, the solar radius is equal to $696192 \pm 247 \mathrm{~km}(\xi)$. The PICARD space-based results as well as PICARD ground-based results show that the solar radius wavelength dependence in the visible and the near-infrared is extremely weak. The differences in inflection point position of the solar radius at $607.1 \mathrm{~nm}, 782.2 \mathrm{~nm}$, and $1025.0 \mathrm{~nm}$ from a reference at $535.7 \mathrm{~nm}$ are less than $60 \mathrm{~km}$ for the different PICARD measurements.
\end{abstract}

Key words. Sun: general - Sun: fundamental parameters - telescopes

\section{Introduction}

The Sun is a star with a radius of $695990 \mathrm{~km}\left(R_{\odot}=959.63 \mathrm{arcsec}\right)$ at one astronomical unit (1 au), which corresponds to the canonical value (Auwers 1891) that is commonly used. This value differs from the nominal solar radius value $\left(R_{\odot}^{N}=695700 \mathrm{~km} \simeq 959.23 \mathrm{arcsec}\right)$ recommended by the XXIXth International Astronomical Union (IAU) General Assembly in 2015 (Prša et al. 2016). The IAU nominal value is based on one transit measurement (Solar Diameter Monitor, Brown et al. 1982) and two helioseismic determinations of the solar radius, in which the solar edge determined from helioseismic data is located below the photosphere. Since the solar radius has a new definition, a correction is required to compare the IAU value $\left(R_{\odot}^{N}\right)$ and the direct measurements of the photospheric solar radius $\left(R_{\odot}\right)$ that are mainly obtained through the inflection point position methods. The difference between the seismic solar radius and the inflection point position is $\sim 333 \mathrm{~km}(\sim 0.46 \mathrm{arcsec})$ for the apparent solar radius at $500 \mathrm{~nm}$ (Haberreiter et al. 2008). Brown \& Christensen-Dalsgaard (1998) have corrected their observations to reduce their radius. Therefore, the direct measurements of the photospheric solar radius $\left(R_{\odot}\right)$ have to be adjusted for comparison.

The absolute determination of the solar radius from direct measurements is a source of discussion even today. Recent results (Table 1) are not consistent and differ with the canonical value of the solar radius $\left(R_{\odot}\right)$. Authors can interpret the discrepancies in relation with the different methods of analysis used in 
A\&A 616, A64 (2018)

Table 1. Recent results of the solar radius at 1 au using different methods and instruments for different wavelength $(\lambda)$.

\begin{tabular}{llllll}
\hline \hline Author(s) & Dates & Method (instrument) & $\lambda(\mathrm{nm})$ & $R_{\odot} \pm \mathrm{STD}(\mathrm{km})$ & $\xi(\mathrm{km})$ \\
\hline Vaquero et al. (2016) & $1773-2006$ & Transit times (several instruments) & - & $695438 \pm 1284$ & - \\
Lamy et al. (2015) & $2010-2015$ & Total solar eclipses (photometers) & 540.00 & $696251 \pm 15$ & - \\
\hline Sofia et al. (2013) & $1992-2011$ & Prism as angular reference (sextant) & 615.00 & $695997 \leqslant R_{\odot} \leqslant 696171$ & \pm 15 \\
\hline Emilio et al. (2012) & 2003 and 2006 & Mercury transits (MDI) & 676.78 & $696345 \pm 65$ & - \\
Hauchecorne et al. (2014) & 2012 & Venus transit (HMI) & 617.30 & $696185 \pm 44$ & - \\
Meftah et al. (2014b) & 2012 & Venus transit (SODISM) & 607.10 & $696156 \pm 44$ & \pm 145 \\
Emilio et al. (2015) & 2012 & Venus transit (HMI) & 617.30 & $695946 \pm 15$ & - \\
\hline IAU $\left(R_{\odot}^{N}\right)$ & 2015 & Transit and helioseismic determinations & - & $\mathbf{6 9 5 7 0 0}$ & - \\
\hline
\end{tabular}

Notes. Solar radius measurements using the transit-timing method, Vaquero et al. (2016) ground-based measurements made in the Royal Observatory of the Spanish Navy; prism angular reference, Solar Disk Sextant (SDS) balloon flights; planet transits, the Michelson Doppler Imager (MDI) on board the Solar and Heliospheric Observatory, the Helioseismic and Magnetic Imager (HMI) on board the Solar Dynamics Observatory, SODISM on board PICARD; and solar eclipses, ground-based measurements made with solar photometers. STD represents the standard error, which depicts the dispersion of individual observations around the mean or median value. $\xi$ is the combined standard uncertainty of the individual standard uncertainties (errors of the uncertainty budget). For example, the typical estimated uncertainty of each SDS measurement (Sofia et al. 2013) is $15 \mathrm{~km}(0.02$ arcsec) with a solar radius that is found to vary by up to $174 \mathrm{~km}$ (real variation and/or error) from 1992 to 2011 .

their work. However, other effects may explain the discrepancies. Mainly, there is the influence of the atmosphere on groundbased measurements and the effects of the space environment on space-based measurements. Lunar and planetary transits of the Sun allow measurements of the solar radius with high accuracy. The determination of a realistic uncertainty budget remains a significant problem and requires special attention in its determination, such as in the analyses of Meftah et al. (2014b). Therefore, it is necessary to distinguish between the combined standard uncertainty of the individual random and systematic uncertainties (based on the uncertainty budget) and the standard error (standard deviation of the median value, or standard error on the fit that allows determining the solar radius). It is also necessary to specify the wavelength in relation to the solar radius measurements. Solar radius measurements at several wavelengths may be useful for testing numerical radiative hydrodynamics simulations of the solar atmosphere.

This work provides new values of the solar radius obtained during the PICARD mission for the space-based and the groundbased segments at several wavelengths. Solar radius observations during the 2012 transit of Venus have been made with the SOlar Diameter Imager and Surface Mapper (SODISM) telescope on board the PICARD spacecraft (Meftah et al. 2014c). In addition, PICARD SOL is the ground-based segment of PICARD (Meftah et al. 2014a), which allows comparison of the solar radius measured in space to those obtained on ground by the PICARD/SODISM II instrument at the Observatoire de la Côte d'Azur (Calern site). One of the PICARD objectives was to determine the absolute values of the solar radius at several wavelengths at $393.37,535.7,607.1,782.2$, and $1025.0 \mathrm{~nm}$. The last four wavelengths were selected for their desired shortage of Fraunhofer absorption lines, while the $393.37 \mathrm{~nm}$ wavelength reflects the chromosphere and activity therein.

PICARD contains a double program with in-space (PICARD spacecraft) and on-ground measurements (PICARD SOL). It aims at perpetuating valuable historical time-series of the solar radius. Space-based observations are a priori more favorable than ground-based observations. However, space-based measurements present technical challenges such as stability of the point spread function (PSF) of the instrument, a harsh operating environment, and a finite mission lifetime. As a reminder, the PICARD spacecraft was launched in June 15, 2010, and was retired in April 2014. On the ground, the instruments are less strongly affected by degradation than in space since maintenance is easy to provide. In addition, if the atmosphere is properly monitored and taken into account, they still represent an opportunity for generating long-term time-series. Groundbased measurements have therefore been carried out by the PICARD/SODISM II instrument beginning in May 2011 and continuing through 2018.

Section 2 provides the most common solar radius definition used in most analyses. Section 3 discusses the influence of the atmosphere on ground-based solar radius measurements. Section 4 shows the results obtained with the PICARD/SODISM II groundbased instrument after making all necessary corrections (angular calibration with stars, Sun distance, refraction, estimation of the turbulence, etc.). The difficulty of ground-based measurements highlights the interest of measurements outside the atmosphere with space-based instruments. Section 5 shows the results obtained with the PICARD/SODISM space-based telescope using the June 2012 transit of Venus (see the image acquired by the SODISM instrument at the end of the transit in Appendix A).

\section{Definition of the solar radius}

The results may be influenced by the definition of the solar radius that is used. The most common solar radius definition is based on the determination of the inflection point position of the solar limb-darkening function (LDF). As explained by Emilio et al. (2015), the absolute value of the solar radius determined by Hauchecorne et al. (2014) cannot be compared with the inflection point position method since the authors adopted a different definition of the solar radius that does not depend on the inflection point position of the LDF. The method of Hauchecorne et al. (2014) is based on the spectral solar radiance decrease estimation in an area around the contact between Venus and the Sun during ingress and egress. Despite this, the SODISM solar radius at $607.1 \mathrm{~nm}$ reported by Meftah et al. (2014b) and by Hauchecorne et al. (2014) with two different methods are very similar, $696156 \pm 145 \mathrm{~km}(959.86 \pm 0.20 \mathrm{arcsec})$ with the inflection point position of the LDF definition and $696149 \pm 138 \mathrm{~km}(959.85 \pm 0.19 \mathrm{arcsec})$ with the spectral solar radiance decrease estimation method. On the other hand, the solar radius obtained during the 2012 transit of Venus by the Helioseismic and Magnetic Imager (HMI) is equal to $695946 \mathrm{~km}$ (959.57 arcsec; Emilio et al. 2015) using the common definition of the solar radius and is equal to $696185 \mathrm{~km}(959.90 \mathrm{arcsec})$ using the Hauchecorne et al. (2014) method. Use of different 
definitions of the solar radius can lead to significantly different results. We here focus only on PICARD results obtained from the common definition of the solar radius (inflection point position of the LDF definition).

For a given solar atmosphere, the solar radius depends on wavelength. The solar atmosphere is primarily composed of the photosphere, the chromosphere, and the corona. The photosphere is the lowest layer of the solar atmosphere. It is about $500 \mathrm{~km}$ thick, providing more than $90 \%$ of the solar emitted radiation (especially in the visible). The height scale of the photosphere is not arbitrary. The base of the photosphere (height of $0 \mathrm{~km}$ ) is defined as the optical depth of one (i.e., $\tau_{500 \mathrm{~nm}}=1$ ). For a given spectral domain, solar atmospheric models allow calculating the solar limb shape and determining the inflection point position with or without Fraunhofer lines. From radiative transfer simulations, we can accept variations of $\sim 18 \mathrm{~km}(\sim 25 \mathrm{mas})$ between measurements made at several wavelengths in the photospheric continuum (Meftah et al. 2014a).

PICARD measurements (space-based and ground-based segments) in the solar photospheric continuum $(535.7 \mathrm{~nm}, 607.1 \mathrm{~nm}$, $782.2 \mathrm{~nm}$, and $1025.0 \mathrm{~nm}$ ) correspond to observations of the photosphere (at a height of between 300 and $400 \mathrm{~km}$ measured from the solar limb level). PICARD also enables observing the chromosphere $(393.37 \mathrm{~nm})$, which is an irregular layer of approximately $1500 \mathrm{~km}$ thickness. The chromosphere extends from 500 to $2000 \mathrm{~km}$ above the photosphere. One of its most prominent spectral line is the CaIIK line, which is split into three regions. Each region shows a specific area of the chromosphere. The formation heights according to Vernazza et al. (1981) are $450-650 \mathrm{~km}$ (at $0.15 \mathrm{~nm}$ from the core, K1), 700-1450 km (K2), and $1800-2000 \mathrm{~km}$ (core at $393.37 \mathrm{~nm}$ in air, K3), where prominences are visible, for instance. The Ca II K line is very sensitive to magnetic activity. However, this line is very wide and complex, showing a profile both in absorption and in emission. It originates in different heights of the chromosphere. The emissive part of this line reflects the temperature of the matter, creating brilliant regions that are called plages or faculae and are mainly situated around sunspots. PICARD Ca II K filters have a wide bandwidth $(\Delta \lambda)$ of $0.7 \mathrm{~nm}$ over the core at $393.37 \mathrm{~nm}$, which covers the entire $\mathrm{K} 1, \mathrm{~K} 2$, and $\mathrm{K} 3$ regions. Thus, PICARD Ca II K imagery reveals magnetic structures of the Sun from about 450 to $2000 \mathrm{~km}$ above the photosphere. Through PICARD observations, we can determine the absolute value of the photospheric and specific "chromospheric" solar radius. Knowledge of the solar radius and its wavelength dependence is of interest for solar physics. These explanations show that it is necessary to evaluate the solar radius wavelength dependence in different wavelength intervals, separating the measurements between the different solar regions: photospheric continuum, and chromosphere (Ca II K observations made by the PICARD team, $\mathrm{H}-\alpha$ observations, etc.).

\section{Influence of the atmosphere on ground-based solar radius measurements}

The accurate determination of the solar radius is strongly influenced by the effects of the atmosphere (refraction and turbulence), particularly for imaging telescopes such as PICARD SOL/SODISM II. Space-based measurements therefore have a high interest in countering atmospheric effects.

\subsection{Influence of atmospheric refraction}

Simulations of atmospheric effects on solar radius measurements show the influence of atmospheric refraction. Refraction is the

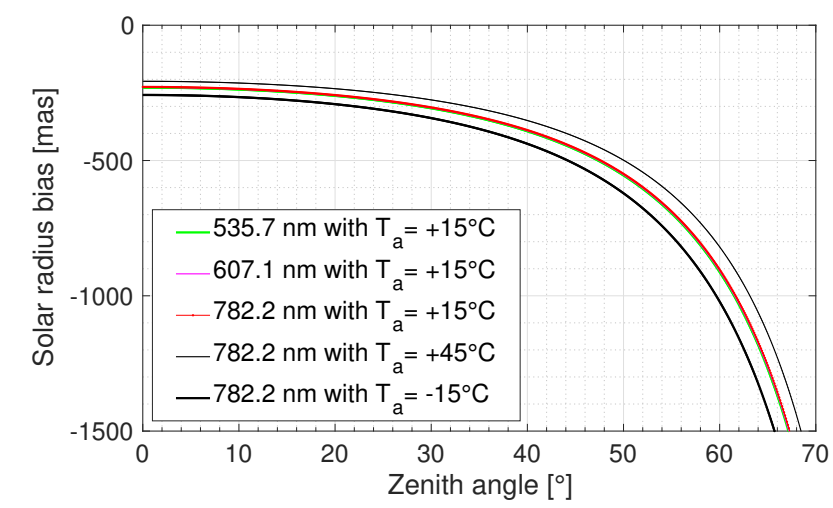

Fig. 1. Impact of refraction on the measurements of the solar radius for several wavelengths. Solar radius bias $\left(R_{0}-R_{\odot}\right)$ or difference between the mean radius of the observed Sun and the true radius of the Sun. The wavelength dependence is weak, in contrast to the air temperature, which has a significant effect on the measurement.

deviation of light from a straight line as it passes through the atmosphere due to the variation in air density as a function of height. The key inputs for atmospheric refraction correction are temperature $\left(T_{\mathrm{a}}\right)$, pressure $\left(P_{\mathrm{a}}\right)$, and relative humidity $\left(f_{\mathrm{h}}\right)$. The solar radius after refraction correction is obtained by

$$
\begin{aligned}
& R_{\odot} \simeq \frac{R_{\mathrm{o}}}{1-k^{\prime}-\frac{1}{2} k^{\prime} \tan ^{2}\left(z_{\odot}^{t}\right)} \\
& k^{\prime}=\alpha(1-\beta)\left[1-\alpha(1-\beta) \sec ^{2}\left(z^{t}\right)\right]
\end{aligned}
$$

where $R_{\mathrm{O}}$ represents the mean observed solar radius, $z_{\odot}^{t}$ is the true zenith angle (from the ephemeris), and $z^{t}$ is the true topocentric zenith angle. The effect of atmospheric refraction is that it changes the true topocentric zenith angle of the Sun to a lower observed angle. $\alpha$ (Eq. (2)) is the air refractivity (Ciddor 1996) for local atmospheric conditions at a given wavelength $(\lambda)$, where $n$ is the refractive index of air. $\beta$ (Eq. (3)) is the ratio between the height $(l)$ of the equivalent homogeneous atmosphere and the Earth radius of curvature $\left(r_{\mathrm{c}}\right)$ at the observer position, assuming an ideal gas law for dry air. For the Calern site, $r_{\mathrm{c}}$ is close to $6367512 \mathrm{~m} . \rho$ is the air density, $g$ is the gravity acceleration, and $C_{\mathrm{a}}$ represents a constant equal to $29.255 \mathrm{~m} \mathrm{~K}^{-1}$ (ideal gas law),

$\alpha=n_{\left(T_{\mathrm{a}}, P_{\mathrm{a}}, f_{\mathrm{h}}, \lambda\right)}-1$

$\beta\left(T_{\mathrm{a}}\right)=\frac{P_{\mathrm{a}}}{\rho \times g \times r_{\mathrm{c}}}=\frac{C_{\mathrm{a}} \times T_{\mathrm{a}}}{r_{\mathrm{c}}}$.

From Eq. (1) we can determine the influence of atmospheric refraction on solar radius measurements as a function of the zenith angle. For this analysis, we took standard conditions of the atmosphere $\left(T_{\mathrm{a}}=15^{\circ} \mathrm{C}, P_{\mathrm{a}}=875 \mathrm{hPa}\right.$, and $\left.f_{\mathrm{h}}=50 \%\right)$. Figure 1 represents the influence of refraction on SODISM II solar radius measurements as a function of the zenith angle. Refraction is much stronger when the Sun is near the horizon than when it is near the zenith. The difference between the mean radius of the observed Sun and the true solar radius is always negative. This simulation highlights the importance of this correction and shows that ground-based measurements $\left(R_{\mathrm{O}}\right)$ will always have smaller observed than true solar radii $\left(R_{\odot}\right)$.

We applied the refraction correction on the mean observed solar radius $\left(R_{\mathrm{O}}\right)$. The correction uncertainty is smaller than 20 mas for zenith distances smaller than $70^{\circ}$. For more accurate measurements, the corrections can be applied individually for each heliographic angle of the solar disk. 


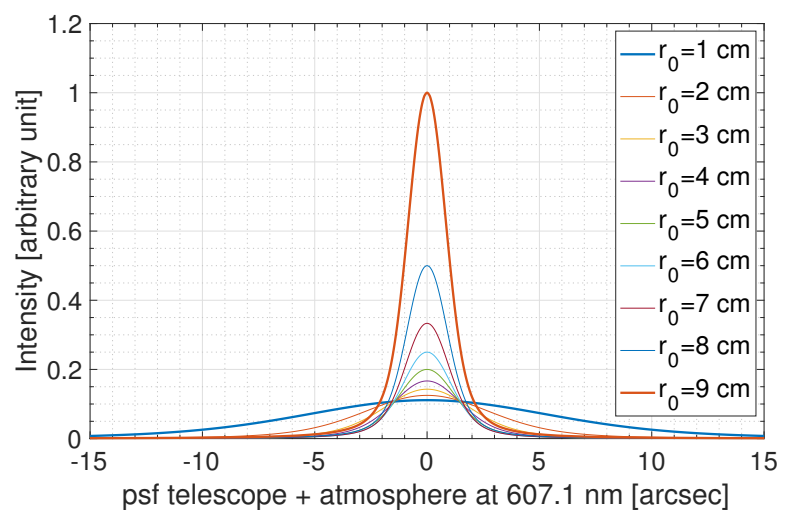

Fig. 2. Impulse response of the instrument-atmosphere system at $607.1 \mathrm{~nm}$ for several values of the Fried parameter $\left(r_{0}\right)$. The instrument parameters used are the focal of the SODISM II instrument $\left(f_{\mathrm{t}}\right)$ of $2.626 \mathrm{~m}$, a pixel size of $13.5 \mu \mathrm{m}$, and a pupil diameter $\left(D_{\mathrm{t}}\right)$ of $9 \mathrm{~cm}$.

\subsection{Influence of atmospheric turbulence}

Measurements of the solar radius made with ground-based instruments are affected by the atmosphere, and particularly by atmospheric turbulence (Roddier 1981). The wavefront of light propagating through the Earth atmosphere is randomly perturbed by turbulence. Simulations of atmospheric effects on solar radius measurements show the influence of seeing conditions. There are three common descriptions of the astronomical seeing conditions at an observatory:

1. The FWHM of the seeing disk (or just "seeing"). A 1 arcsec seeing is good for astronomical sites.

2. The Fried (1965) parameter $\left(r_{0}\right)$ and the timescale $\left(\tau_{0}\right)$. The Fried parameter is the diameter of the coherence area of the degraded wavefront. It also corresponds to the diameter of an equivalent telescope whose spatial resolution results from turbulence effects (Roddier 1981). For the Calern site, the median Fried parameter is on the order of $3.3 \mathrm{~cm}$ at $535.7 \mathrm{~nm}$ (Ikhlef et al. 2016) in one year of observations. Irbah et al. (2016) highlighted lower $r_{0}$ values for few days of observations with a non-optimized instrument. $\tau_{0}$ is the timescale over which the changes in the turbulence become significant.

3. The turbulence profile as a function of altitude $\left(C_{n}^{2}(h)\right)$.

Other parameters characterize atmospheric turbulence and are listed below.

1. The outer scale vertical profile $\left(L_{0}(h)\right)$, which is the distribution of the characteristic scale of the largest velocity inhomogeneities in turbulent layers.

2. The spatial coherence outer scale $\left(\mathcal{L}_{0}\right)$, which defines the maximum size of wavefront perturbations that remain coherent. For the Calern site, the outer scale median value is $\sim 6.3 \mathrm{~m}$ (Ikhlef et al. 2016).

3 . The isoplanatic patch $\left(\theta_{0}\right)$, which represents the angle where phase or speckles remain correlated.

A PICARD SOL turbulence monitor (Moniteur d'Images Solaires Franco-Algérien, MISOLFA) was designed (Assus et al. 2002) to provide estimates of turbulence parameters $\left(r_{0}, \mathcal{L}_{0}, \theta_{0}, \tau_{0}\right.$, and $\left.C_{n}^{2}(h)\right)$. This work is still in progress (Irbah et al. 2010; Ikhlef et al. 2016) to obtain not only daily and monthly averages, but continuous estimates of these parameters.

We developed a simple model, as shown in Appendix B, to highlight the strong influence of atmospheric turbulence on the determination of the solar radius with ground-based telescopes. This approach demonstrates that the main source of uncertainty
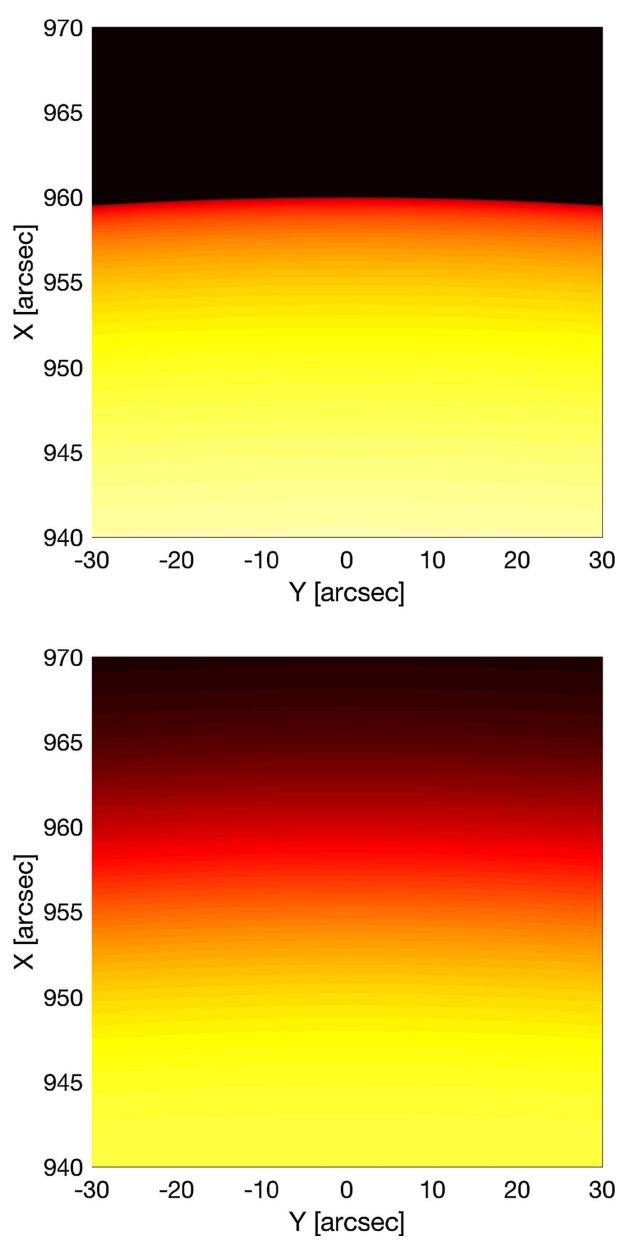

Fig. 3. Top: image of the Sun made using the instrument-atmosphere system at $607.1 \mathrm{~nm}$ with $r_{0}=9 \mathrm{~cm}$. Bottom: at $607.1 \mathrm{~nm}$ with $r_{0}=1 \mathrm{~cm}$.

and bias for all ground-based astrometry programs is the effect of atmospheric turbulence.

\subsubsection{Impact of the atmospheric turbulence on solar radius}

At the Calern site, the Fried parameter can vary between $1 \mathrm{~cm}$ and up to $6 \mathrm{~cm}$ during SODISM II images acquisitions. Figure 2 shows the impulse responses of the instrument-atmosphere system for different values of the Fried parameter. The atmospheric turbulence causes a spread and blur of the solar limb when $r_{0} \rightarrow 1 \mathrm{~cm}$ (Fig. 3). This causes a displacement of the position of the inflection point of the limb-darkening function (Fig. 4). For this analysis, we can use the model of the solar limb-darkening function (HM98) developed by Hestroffer \& Magnan (1998) or the Neckel 2005 model (Neckel 2005), which represents the physical solar limb better. Figure 5 shows the impact of the atmospheric turbulence on the solar radius determination at several wavelengths.

\subsubsection{Conclusions}

The main problem is estimating the optical transfer function (OTF), which describes atmospheric turbulence effects on recorded images. Several procedures have been developed a posteriori on observations to calculate the instrument-atmosphere system PSF. This simulation highlights the importance of the turbulence correction and shows that ground-based measurements will always have smaller solar observed radii. For telescopes with 
M. Meftah et al.: Extremely weak solar radius wavelength dependence in the visible and the near-infrared
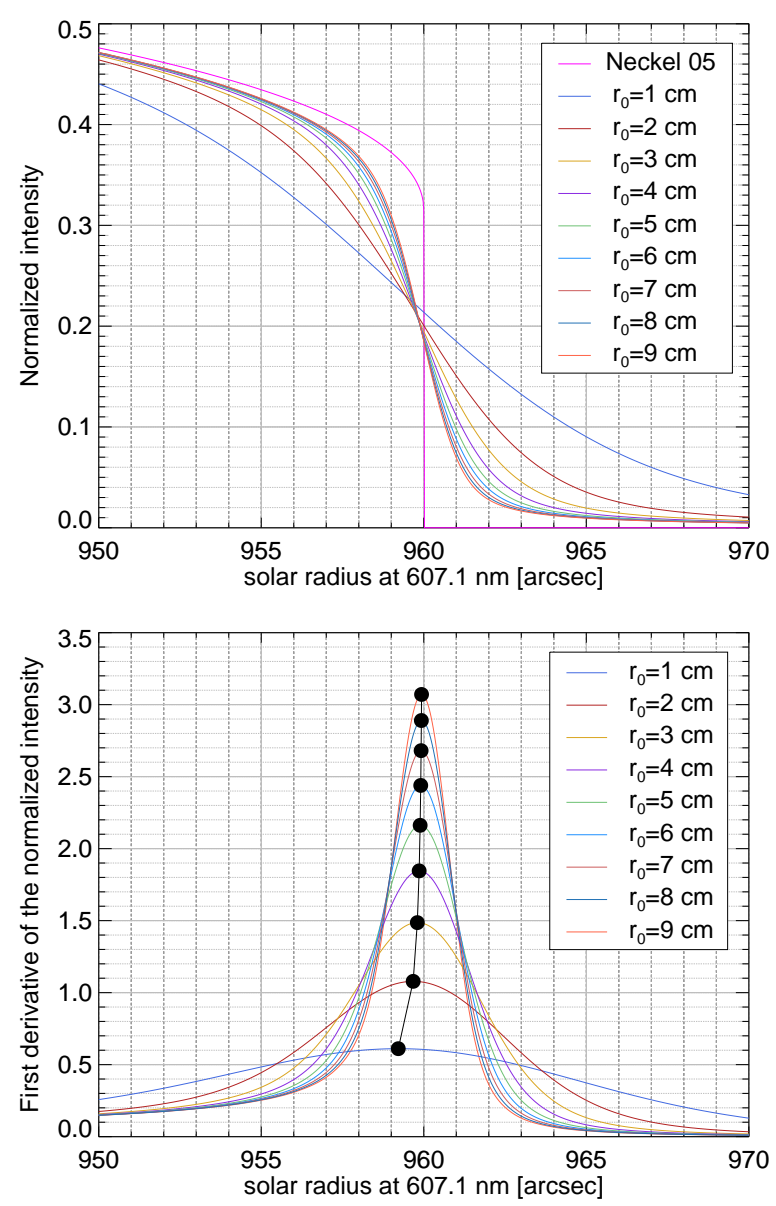

Fig. 4. Top: solar limb-darkening functions $\left(\mathrm{LDF}_{\mathrm{t}}\right.$ at $\left.607.1 \mathrm{~nm}\right)$ obtained with several values of the Fried parameter compared to the Neckel 2005 empirical solar model without atmospheric turbulence. Bottom: first derivative of the solar limb-darkening functions $\left(\mathrm{LDF}_{\mathrm{t}}\right.$ at $\left.607.1 \mathrm{~nm}\right)$ and location of the inflection point position of the solar limb-darkening function (observed solar radius).

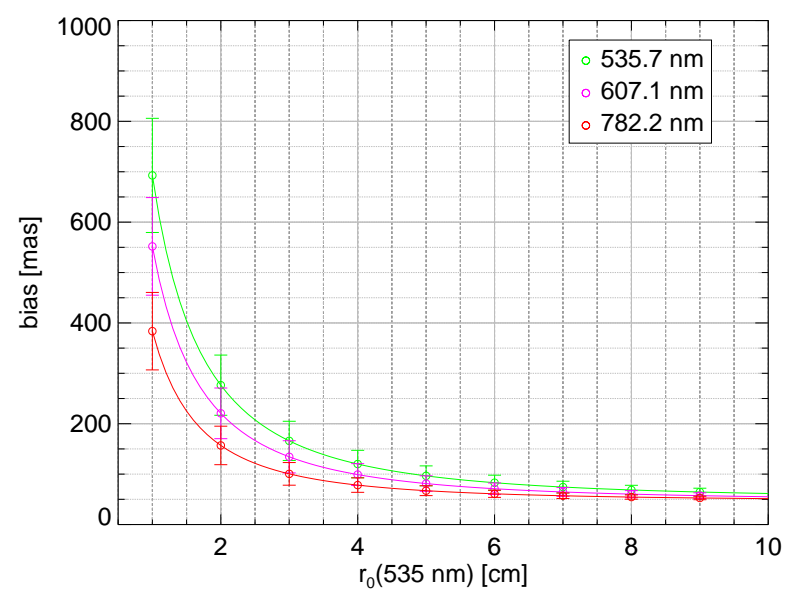

Fig. 5. Impact of the atmospheric turbulence on the solar radius determination $(535.7,607.1$, and $782.2 \mathrm{~nm}$ ) for a perfect instrument (theoretical PSF). Solar radius bias $\left(R_{\odot}-R_{\mathrm{o}}\right)$ or difference between the true radius of the Sun and the mean radius of the observed Sun as a function of the Fried parameter $\left(r_{0}\right.$ at $\left.535.7 \mathrm{~nm}\right)$. The bottom of the error bar corresponds to $\mathcal{L}_{0}=1 \mathrm{~m}$, the top of the error bar corresponds to the Kolmogorov model $\left(\mathcal{L}_{0} \rightarrow \infty\right)$. diameters $\left(D_{\mathrm{t}}\right)$ larger than $r_{0}$, the image resolution is determined primarily by the atmosphere. The accurate determination of the solar radius from ground-based data is not obvious and may introduce significant uncertainties in final results.

\section{Solar radius determined from PICARD/SODISM II ground-based measurements}

The SODISM II solar radius results (photospheric continuum and "chromospheric"), corrected as described in Meftah et al. (2014a), are listed in Table 2.

At the end of life of the PICARD SOL mission, a laboratory characterization of the SODISM 2 instrument (PSF and plate scale) will be carried out in order to refine the results. A test bench with a xenon lamp and a collimator will be used to create an artificial image of the Sun. An optical distortion target (pinholes) will be placed between the collimator and the lamp to characterize the telescope. Moreover, the MISOLFA data analysis will be improved $\left(r_{0}, \mathcal{L}_{0}\right.$, etc. $)$.

\subsection{Solar radius wavelength dependence}

The PICARD SOL solar radius at $393.37 \mathrm{~nm}$ (Fig. 6) is largely higher than the curve fitting proposed by Rozelot et al. (2015). This result shows that it is interesting to evaluate the solar radius wavelength dependence by properly taking into account different solar regions before fitting polynomial functions to the measurements.

The SODISM II solar radius dependence on the wavelength in the visible and the near-infrared is extremely weak, as shown in Fig. 6. The poor agreement with the curve fitting proposed by Rozelot et al. (2015) is evident. Moreover, Rozelot et al. (2015) investigated the solar radius wavelength dependence in different wavelength intervals by fitting polynomial functions to the measurements in the near-ultraviolet, the near-infrared, and the visible from different ground-based instruments. In their analysis, they considered the SODISM II solar radius obtained at 535.7 and $607.1 \mathrm{~nm}$, but did not take into account the solar radius obtained at 782.2 and $1025.0 \mathrm{~nm}$, although it was published in Meftah et al. (2014a). The inclusion of these results significantly changes their conclusions. The differences of the inflection point position $(\triangle I P P)$ at $607.1 \mathrm{~nm}, 782.2 \mathrm{~nm}$, and $1025.0 \mathrm{~nm}$ from the inflection point position reference at $535.7 \mathrm{~nm}$ are indeed smaller than 100 mas for the different SODISM II measurements. Moreover, Meftah et al. (2014a) analyzed the impact of an instrumental effect (optical aberration) on the inflection point position. They demonstrated that the $\Delta$ IPP differences are dominated by a poor correction of the wavelength dependence of instrumental (plate-scale, PSF) and atmospheric effects and not by the weak wavelength dependence of the solar LDF itself in the photospheric continuum. Therefore, the solar radius wavelength dependence in the visible and the near-infrared is extremely weak, and still lies within the 100-200 mas combined observational uncertainties.

\subsection{Discussion of the combined standard uncertainty (uncertainty budget) and standard error (standard deviation of the solar radius median value)}

Rozelot et al. (2015) summarized the solar radius observations at several wavelengths and provided errors on the determination of the solar radius for each experiment, although they are not of the same nature. For the PICARD case, the "error" on the solar radius 
Table 2. Summary of the PICARD solar radius observations at several wavelengths.

\begin{tabular}{llllllll}
\hline \hline$\lambda(\mathrm{nm})$ & $\Delta \lambda(\mathrm{nm})$ & ${\text { Radius at } 1 \mathrm{au}^{a}}$ & STD $(\operatorname{arcsec})$ & $\xi[\operatorname{arcsec}]$ & $R_{\odot}(\mathrm{km})$ & Experiment & References \\
\hline 393.37 & 0.7 & $961.360 \operatorname{arcsec}$ & \pm 0.106 & \pm 0.387 & $697244 \pm 281^{b}$ & SODISM II & This work \\
535.7 & 0.5 & $959.777 \operatorname{arcsec}$ & \pm 0.125 & \pm 0.194 & $696096 \pm 141^{b}$ & SODISM II & Meftah et al. (2014a) \\
607.1 & 0.7 & $959.862 \operatorname{arcsec}$ & \pm 0.126 & \pm 0.185 & $696157 \pm 134^{b}$ & SODISM II & Meftah et al. (2014a) \\
782.2 & 1.6 & $959.876 \operatorname{arcsec}$ & \pm 0.099 & \pm 0.129 & $696168 \pm 94^{b}$ & SODISM II & Meftah et al. (2014a) \\
1025.0 & 6.4 & $959.832 \operatorname{arcsec}$ & \pm 0.181 & \pm 0.105 & $696136 \pm 76^{b}$ & SODISM II & Meftah et al. (2014a) \\
\hline \hline 535.7 & 0.5 & $959.83 \operatorname{arcsec}$ & \pm 0.15 & \pm 0.36 & $696134 \pm 261$ & SODISM & This work \\
$\mathbf{6 0 7 . 1}$ & $\mathbf{0 . 7}$ & $\mathbf{9 5 9 . 8 6}$ arcsec & $\pm \mathbf{0 . 0 6}$ & $\pm \mathbf{0 . 2 0}$ & $\mathbf{6 9 6 1 5 6} \pm \mathbf{1 4 5}$ & SODISM & Meftah et al. (2014c) \\
782.2 & 1.6 & 959.91 arcsec & \pm 0.19 & \pm 0.34 & $696192 \pm 247$ & SODISM & This work \\
\hline
\end{tabular}

Notes. STD represents the standard deviation of the median value $(\sigma=1) . \xi$ represents the combined standard uncertainty of the individual standard uncertainties (based on the uncertainty budget). $\Delta \lambda$ corresponds to the bandwidth of the central wavelength $(\lambda)$ of the filter. ${ }^{(a)}$ The solar radius is based on the determination of the inflection point position. ${ }^{(b)}$ The PICARD/SODISM II results are obtained from an estimate of the Fried parameter $\left(r_{0}\right)$. The PICARD/SODISM II combined uncertainties $(\xi)$ may be undervalued compared to those of SODISM, who are overestimated (center detections and PICARD/SODISM on-board timing).

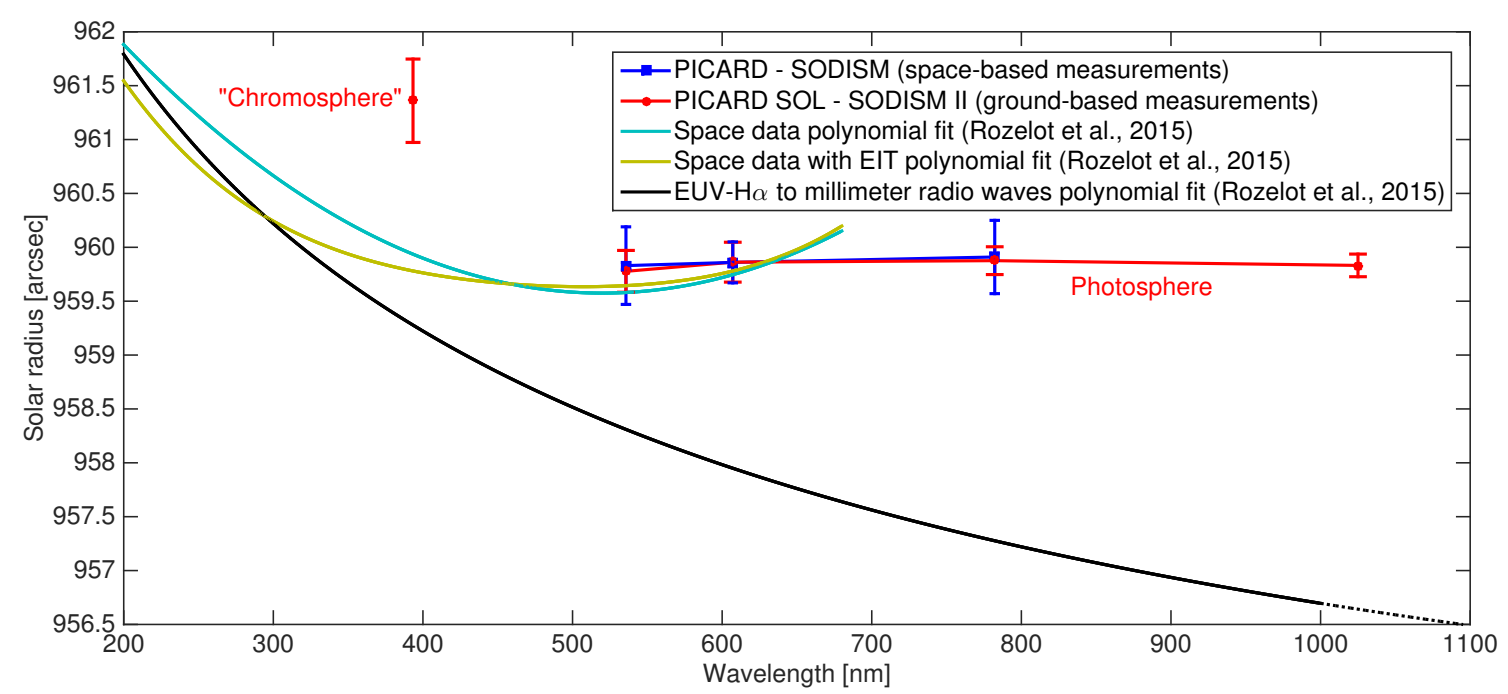

Fig. 6. Solar radius variation with wavelength from PICARD SOL ground-based data (SODISM II data in red) and from PICARD spacecraft data (SODISM data in blue). The "chromospheric point" corresponds to the "PICARD" Ca II K line band. We also plot the solar radius variations with wavelength from data polynomial fits (Rozelot et al. 2015).

determination corresponds to a combined standard uncertainty of the individual standard uncertainties $(\xi)$ and not to a standard deviation of the solar radius median value (STD). The error is the difference between the measured value and the "true" value of the variable being measured, and the uncertainty is a quantification of the doubt about the measurement result. PICARD results are associated with an uncertainty budget with random (when repeating the measurement gives a randomly different result) and systematic (when the same influence affects the result for each of the repeated measurements) uncertainty sources.

\section{Solar radius determined from PICARD/SODISM space-based measurements}

The effects of the space environment (ultraviolet (UV), polymerization, contamination, radiation, South Atlantic Anomaly, thermal cycling, etc.) lead to considerable degradation of the instruments in orbit, such as PICARD/SODISM (Meftah et al. 2014c), which directly observed the Sun from July 2010 to March 2014. All these effects require complex corrections (optical, thermal, and electrical). The best way is to determine the solar radius during a planetary transit to counter calibration errors and aging in space due to the effects of the space environment. We therefore used the transit of Venus as an absolute calibration to determine the solar radius accurately at several wavelengths.

\subsection{Method implemented for PICARD/SODISM data}

This section describes the method used to determine the solar radius accurately at several wavelengths from PICARD/SODISM observations. The 2012 transit of Venus is used to provide an absolute plate scale to calibrate the solar inflection point position. Our results are based on the determination of the inflection point position of the solar limb-darkening function. The true solar radius $\left(R_{\odot}\right)$ at a given wavelength is obtained from determining several parameters: the plate scale of the telescope $(\operatorname{PS}(\theta)$ in $\operatorname{arcsec}$ per pixel), the optical distortion function of the telescope $\left(F_{d}^{t}(\theta)\right.$ without units) and the mean solar radius of the normalized image at one astronomical unit $\left(\left\langle R_{\text {image }}^{\mathrm{PS}}\right\rangle\right.$ in pixels $)$. The direct measurements of the photospheric solar radius $\left(R_{\odot}\right)$ are obtained from Eq. (4). For each image acquired during the 2012 transit of Venus, the mean solar radius at a given wavelength is determined by

$R_{\odot}=\left\langle R_{\mathrm{image}}^{\mathrm{PS}}\right\rangle \times \operatorname{PS}(\theta) \times F_{\mathrm{d}}^{t}(\theta)$. 
The plate scale of the telescope is obtained from the determination of the positions of the Sun and Venus from ephemeris and PICARD/SODISM images (Eq. (5)). It is necessary to know the ephemeris and to calculate the positions of the Sun and Venus from images taken by the SODISM instrument. The transit of Venus is a very convenient tool for calibrating the SODISM telescope (PSF and plate scale),

$$
\operatorname{PS}(\theta)=\frac{d_{\odot \uparrow}^{E}}{\sqrt{\left(X_{\odot}^{I}-X_{\odot}^{I}\right)^{2}+\left(Y_{\odot}^{I}-Y_{\odot}^{I}\right)^{2}}}=\frac{d_{\odot \uparrow}^{E}}{d_{\odot \odot}^{I}},
$$

where $d_{\odot \odot}^{E}$ corresponds to the distance between the Sun and Venus obtained from the ephemeris ( $\operatorname{arcsec}), X_{\odot}^{I}$ and $Y_{\odot}^{I}$ correspond to the Sun positions obtained from PICARD images (pixels), and $X_{\odot}^{I}$ and $Y_{q}^{I}$ correspond to the Venus positions obtained from PICARD images (pixels).

The optical distortion function was obtained during a PICARD/SODISM calibration (determination of the telescope distortion during spacecraft rotation using a total of 1040 images at a given wavelength). During distortion measurements campaigns, the PICARD spacecraft revolves around the PICARDSun axis by steps of $30^{\circ}$ from North to West (clockwise). Solar images are taken for each roll step of the spacecraft (80 images per roll step). The optical distortion function is obtained by

$$
F_{\mathrm{d}}^{t_{\mathrm{d}}}(\theta)=\frac{R_{\text {image }}^{t_{\mathrm{d}}}(\theta)}{\left\langle R_{\text {image }}^{t_{\mathrm{d}}}\right\rangle},
$$

where $\left\langle R_{\text {image }}^{t_{\mathrm{d}}}\right\rangle$ (in pixels) represents the value of the mean solar radius obtained during the distortion campaign and $R_{\text {image }}^{t_{\mathrm{d}}}(\theta)$ corresponds to the radius value (in pixels) at the desired angular position $(\theta)$. The optical distortion function is obtained from many images acquired at several angular positions of the spacecraft. Thus, the random-type error associated with its determination is very weak (a few mas).

\subsubsection{Determination of the positions of the Sun and Venus from ephemeris}

The ephemeris calculation requires knowledge of the spacecraft positions, the Venus positions, and the Sun positions in the J2000 coordinate system. The PICARD spacecraft coordinates were corrected for precession (Capitaine et al. 2003), nutation (Seidelmann 1992), and aberration due to the speed of the spacecraft. The uncertainty obtained is 0.1 mas. Planetary theories (Fienga et al. 2008) were used to determine the positions of Venus and the Sun. The uncertainty obtained is 5 mas. These uncertainties can be considered negligible compared to the uncertainty associated with the PICARD on-board timing ( 80 mas), which could be significantly reduced (work in progress). For each PICARD date of image, the distance between the center of the Sun and the center of Venus was calculated using the ephemeris $\left(d_{\odot}^{E}\right.$ in $\left.\operatorname{arcsec}\right)$.

\subsubsection{Determination of the positions of the Sun and Venus from PICARD/SODISM images}

The main uncertainty depends on the determination of the position of the Sun and Venus (up to a few tenths of a pixel). These positions were determined for each image taken by the SODISM
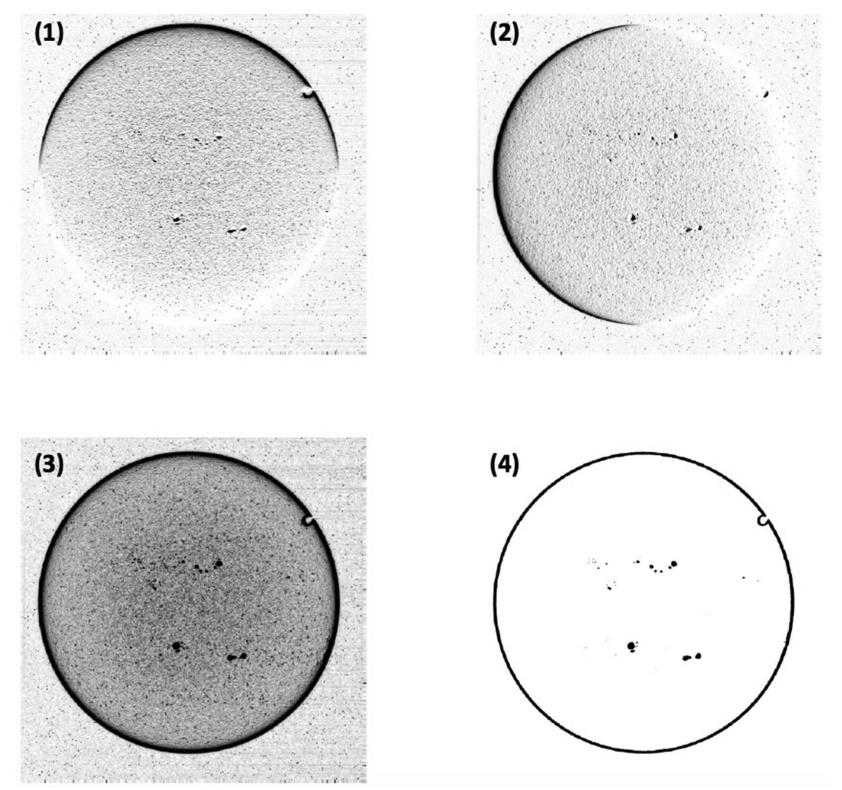

Fig. 7. Panel 1: PICARD/SODISM image $(607.1 \mathrm{~nm})$ acquired on June 6, 2012 at 04H31 UTC with a horizontal gradient. Panel 2: vertical gradient. Panel 3: norm of the gradient. Panel 4: norm of the gradient with the use of a threshold.

instrument. The distance between the center of the Sun and the center of Venus was calculated ( $d_{\odot \odot}^{I}$ in pixels).

This section describes the method used to obtain the parameters used in the determination of the plate scale (radius and location of the center of the Sun and Venus). Six steps are necessary:

1. Noise removal. The image is processed with a median filter to remove outlier pixels. Then, a Gaussian blur is applied to smooth the edges in the image. The displacement of Venus across the solar disk generates a slight blur.

2. Extraction of contours. The contours are extracted using the Sobel filter (operator used in image processing for the detection of gradients) and the Canny method (a tool that allows good contour detection and good localization). A threshold applied to the norm of the gradient is used to select the pixels of the contours. An example of gradient extraction on one image is shown in Fig. 7 using the image of the Sun acquired by the PICARD/SODISM instrument at $607.1 \mathrm{~nm}$ in June 2012.

3. Center detection using the Hough method. The Hough method (Hough 1962; Duda \& Hart 1972) is used in image segmentation; this method is not very sensitive to noise and allows detecting geometrical shapes for a given image. This method is used to detect the centers of the Sun and Venus. This is a good approximation to detect the centers in order to start the extraction of each inflection point position of the contours.

4. Extraction of the inflection point position. The next step is to extract a set of edge points from the contour of the Sun and Venus. This is done by analyzing the profile whose abscissa is a range of radii and whose ordinates are the intensity levels interpolated along radial segments. The edge of the contour is determined by the abscissa for which the above profile reaches the highest gradient. This abscissa can be computed with three methods: an analysis of the numerical first derivative, an adjustment of the profile by a polynomial function, and an adjustment of the profile by a rational function. We employed the first method.

5. Characterization of the ellipse. After the edge points are computed, the set of points is fitted by a circle, a non-oriented 
ellipse, or an oriented ellipse. The ellipse center is computed and the edge points are extracted again using the new center to extract a new set of profiles. This new set of profiles is used to compute a new ellipse whose center is used to extract another new set of profiles, and so on until the distance between two consecutive centers is lower than the value (in pixels) of a stop criterion. This refinement should converge to a best characterization of the ellipse because the radial segments are becoming more and more perpendicular to the tangents to the ellipse contours, and the edges consequently become ever more accurate (the gradients are increasingly higher) in the profile curves.

6. Average radius of the Sun and Venus. The apparent radii of the Sun and Venus are determined from the position of the different points of the contours (median value).

\subsection{Results}

The PICARD/SODISM solar radius results (photospheric continuum at 535.7, 607.1, and 782.2 nm), using the method described in Sect. 5.1, are listed in Table 2. At 215.00 and $393.37 \mathrm{~nm}$, we have no results because the SODISM images are blurred and no distortion correction is available around the period time of the transit of Venus in 2012. A realistic uncertainty budget (Table 3 ) is provided for each solar radius obtained with the SODISM space-based telescope during the 2012 Venus transit. The uncertainty budget considers several sources of errors (detection of Venus and Sun centers from PICARD images, Sun and Venus positions from ephemeris (planetary theory), PICARD on-board timing, PICARD spacecraft position, and optical distortion correction from images). For each result, we calculated the combined standard uncertainty of the individual standard uncertainties (based on the uncertainty budget) and the standard error (standard deviation of the median value).

The value of the solar radius at $607.1 \mathrm{~nm}$ is equal to $959.86 \pm 0.2 \operatorname{arcsec}(\xi)$. This result corresponds to the most accurate value obtained with the PICARD/SODISM data (higher number of measurements, measurements during contacts, and the best PSF). The standard deviation of the median value is \pm 0.06 arcsec (STD). The combined standard uncertainty $(\xi)$ takes into account the different types of errors (random and systematic). From the method developed in Sect. 5.1, we calculate the solar radius at $535.7 \mathrm{~nm}$ and at $782 \mathrm{~nm}$. For each image at a given wavelength $(535.7$ and $782.2 \mathrm{~nm})$ during the June 2012 transit of Venus, we determined the mean value of the solar radius and its center in this way. From the knowledge of the plate scale $\left(\operatorname{arcsec}\right.$ pixel $\left.^{-1}\right)$, we can calculate the value of the solar radius (arcsec) at 1 au (Fig. 8, curves plotted with black squares). The new values obtained at 535.7 and $782.2 \mathrm{~nm}$ can be corrected for the optical distortion function of the telescope $\left(F_{d}^{t}(\theta)\right)$. The plate scale depends on its position from the center to the limb (distance $r$ in $\mathrm{mm}$ on the charge-coupled device (CCD)). The Ritchey-Chrétien configuration of the SODISM telescope minimizes spherical and coma aberrations, but this design presents some field curvature. Moreover, since the image quality was primarily required at the solar limb, the CCD was consequently positioned to reach its best focus there, that is, about 16 arcmin away from the field-of-view center. Figure 8 shows the corrected measurements (distortion correction) at each wavelength (535.7, 607.1, and 782.2 nm) near the solar limb when $r$ is between 8.5 to $11.5 \mathrm{~mm}$. At $535.7 \mathrm{~nm}$, the solar radius is equal to $959.83 \pm 0.36 \operatorname{arcsec}(\xi)$, that is, consistent with the results obtained at $607.1 \mathrm{~nm}$. The standard deviation of the solar radius median value (standard error) is \pm 0.15 arcsec because we only
Table 3. Error budget in the determination of the PICARD/SODISM solar radius (typical values in mas) with error types (random (R) or systematic (S)).

\begin{tabular}{llll}
\hline \hline Error source (error type) & $535.7 \mathrm{~nm}$ & $607.1 \mathrm{~nm}$ & $782.2 \mathrm{~nm}$ \\
\hline Center of Venus (R) & 348 & 178 & 326 \\
Center of the Sun (R) & 14 & 11 & 16 \\
Planetary theory (S) & 5 & 5 & 5 \\
PICARD on-board timing (R) & 80 & 80 & 80 \\
Spacecraft position (R) & 0.1 & 0.1 & 0.1 \\
Distortion correction (R) & 10 & 5 & 10 \\
\hline$\xi$ (combined uncertainty) & 362 mas & $201 \mathrm{mas}$ & $341 \mathrm{mas}$ \\
\hline
\end{tabular}

Notes. The uncertainty budget is an aid for specifying the expanded measurement uncertainty. The individual measurement uncertainty factors are summarized, usually in tabular form, in the measurement uncertainty budget. $\xi$ is the combined standard uncertainty.

have few images near the solar limb. Similarly, at $782.2 \mathrm{~nm}$, the solar radius is equal to $959.91 \pm 0.34 \operatorname{arcsec}(\xi)$. The standard deviation is \pm 0.19 arcsec (few images).

Therefore, the SODISM solar radius as a function of wavelength in the visible $(535.7 \mathrm{~nm}$ and $607.1 \mathrm{~nm})$ and the nearinfrared $(782.2 \mathrm{~nm})$ is extremely weak, as shown in Fig. 6. The PICARD photospheric solar radius depending on the wavelength is almost constant, in contrast to the claim made in Rozelot et al. (2015). Our results using the Venus transit as an absolute calibration clearly show the dependence of the solar radius on wavelength, which raised many questions.

\section{Conclusions}

One of the best methods to determine the solar radius accurately is to measure it during planet transits (Venus or Mercury). The planet transits can even be used as an absolute calibration to determine the solar radius accurately. This allows countering calibration errors, effects of the atmosphere for ground-based instruments, and aging in space for space-based instruments.

From the PICARD mission, we obtained the solar radius at several wavelengths and from different solar atmosphere regions. PICARD data show that the solar radius wavelength dependence in the visible and the near-infrared is extremely weak. The differences of the inflection point position of the solar radius at $607.1 \mathrm{~nm}, 782.2 \mathrm{~nm}$, and $1025.0 \mathrm{~nm}$ from a reference at $535.7 \mathrm{~nm}$ are smaller than 100 mas for the different PICARD measurements. The photospheric solar radius dependence on the wavelength is extremely weak, in contrast to claim made in Rozelot et al. (2015), and this is even expected since the density profile of the Sun near the photosphere is very sharp.

Rozelot et al. (2015) claimed that the solar radius wavelength dependence were clear. From X-ray to radio waves, they analyzed the solar radius dependence on wavelength using data sets that are associated with scientific publications. For measurements in the visible and the near-infrared, their analysis requires an improvement. Our work sheds new light on the solar radius wavelength dependence. The PICARD data showed that the solar radius wavelength dependence in the visible and the near-infrared is extremely weak. Moreover, it is necessary to distinguish the wavelength dependencies of the solar radius based on two different regions of the solar atmosphere (photosphere and chromosphere). It is known that the Ca II K line (the K3 region lies at $393.37 \mathrm{~nm}$ ) originates in the lower part of the chromosphere and the $\mathrm{H} \alpha$ line (core at $656.28 \mathrm{~nm}$ ) in the mid- 

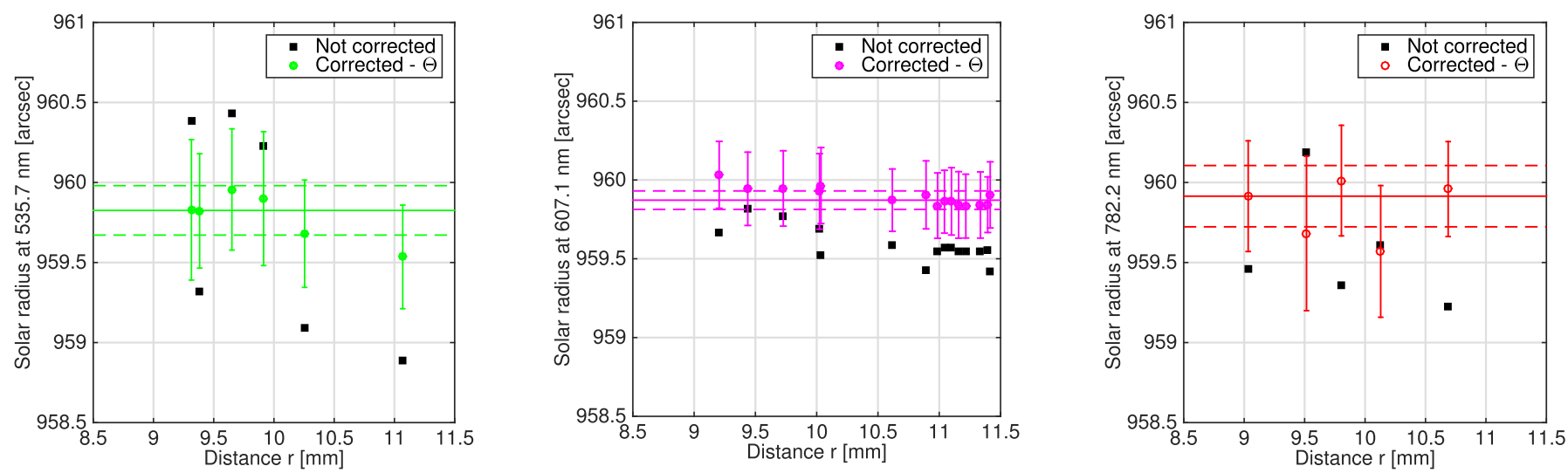

Fig. 8. Solar radius measurements $(535.7,607.1$, and $782.2 \mathrm{~nm})$ obtained from PICARD/SODISM measurements during the Venus transit. The solar radius at $1 \mathrm{au}$ (no distortion correction) is plotted with black squares, and the solar radius at 1 au (with distortion correction) is plotted with circles. $r$ represents the distance from the center of the Sun image on the SODISM CCD to the solar limb $(\sim 11.5 \mathrm{~mm})$. We are particularly interested in the values at the solar limb; the PICARD/SODISM telescope is focused on this position.

dle chromosphere. The $\mathrm{H} \alpha$ core region $(1500 \mathrm{~km}$ above the photosphere) is lower than the $\mathrm{K} 3$ region $(1800 \mathrm{~km})$. Thus, when evaluating the solar radius wavelength dependence in different wavelength intervals, one needs to consider these differences before fitting any polynomial functions to the measurements. The PICARD/SODISM II solar radius at $393.37 \mathrm{~nm}(\Delta \lambda=0.7 \mathrm{~nm})$ is $\sim 1500 \mathrm{~km}$ above the base of the photosphere.

Correcting the PICARD/SODISM solar radius value (at $535.7 \mathrm{~nm})$ of $696134 \pm 261 \mathrm{~km} \quad(959.83 \pm 0.36 \mathrm{arcsec})$ by $\sim 333 \mathrm{~km}(\sim 0.46 \operatorname{arcsec})$ for the apparent solar radius at $500 \mathrm{~nm}$, our corrected results $(695801 \pm 261 \mathrm{~km} ; 959.37 \pm 0.36 \operatorname{arcsec})$ from the space-based instrument are fully consistent with the nominal solar radius adopted by the IAU $(695700 \mathrm{~km}$ $\left(959.23\right.$ arcsec) with respect to $\left.\tau_{\text {Ross }}=2 / 3\right)$. These results confirm the reconciliation between the inflection point measurements and the seismic radii within the combined uncertainties $(\xi)$, as demonstrated in this analysis.

Our most reliable and accurate result is obtained with the PICARD/SODISM space-based instrument at $607.1 \mathrm{~nm}$. The PICARD solar radius $\left(R_{\odot}^{P}\right)$ is $695823 \pm 145 \mathrm{~km}$ $(959.40 \pm 0.20 \mathrm{arcsec} ; \xi)$ using the IAU definition of the solar radius, where $R_{\odot}^{N}=695700 \mathrm{~km}$. The standard deviation of the $R_{\odot}^{P}$ solar radius median value is $\pm 44 \mathrm{~km}$. We find a PICARD solar radius value $\left(R_{\odot}^{P}\right)$ higher than that of the $\operatorname{IAU}\left(R_{\odot}^{N}\right)$. The value adopted by the IAU is defined by the layer where the optical

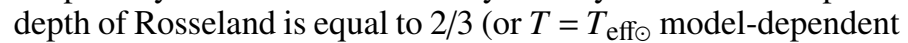
definition). The value of Haberreiter et al. (2008) is based on two helioseismic estimates (f modes; and not three) and one transit measurement (SDM) that was previously corrected to be reduced to $T=T_{\text {eff } \odot}$. The third measurement, which should not be there, decreases the average value significantly. The IAU value may therefore be considered underestimated, which is in line with the result we propose from PICARD analysis as a reference.

The PICARD/SODISM analysis provides a result of the solar radius with a realistic uncertainty budget and a combined standard uncertainty of the individual standard uncertainties $(\xi)$. The error derived from the PICARD on-board timing is certainly overestimated. Recent analyses showed that the on-board timing is close to zero for differential measurements during the Venus transit $(\sim 6 \mathrm{~h})$, which may reduce the combined uncertainty from \pm 145 to $\pm 132 \mathrm{~km}$ at $607.1 \mathrm{~nm}$.

Acknowledgements. This work was supported by Programme National SoleilTerre (PNST, France) and CNES (Centre National d'Études Spatiales, France).
The developing institutes of the PICARD SOL mission and PICARD/SODISM are LATMOS and OCA. This work is dedicated to the memory of Mireille Meissonnier, who was a key person at the beginning of the PICARD program in LATMOS (formerly Service d'Aéronomie du CNRS). The authors would also like to thank the referee for their valuable comments and suggestions that improved the quality of the manuscript.

\section{References}

Assus, P., Borgnino, J., Martin, F., et al. 2002, in Astronomical Site Evaluation in the Visible and Radio Range, eds. J. Vernin, Z. Benkhaldoun, \& C. MuñozTuñón, ASP Conf. Ser. 266, 134

Auwers, A. 1891, Astron. Nachr., 128, 361

Brown, T. M., \& Christensen-Dalsgaard, J. 1998, ApJ, 500, L195

Brown, T. M., Elmore, D. F., Lacey, L., \& Hull, H. 1982, Appl. Opt., 21, 3588

Capitaine, N., Wallace, P. T., \& Chapront, J. 2003, A\&A, 412, 567

Ciddor, P. E. 1996, Appl. Opt., 35, 1566

Duda, R. O., \& Hart, P. E. 1972, Commun. Assoc. Comp. Machin., 15, 11

Emilio, M., Kuhn, J. R., Bush, R. I., \& Scholl, I. F. 2012, ApJ, 750, 135

Emilio, M., Couvidat, S., Bush, R. I., Kuhn, J. R., \& Scholl, I. F. 2015, ApJ, 798, 48

Fienga, A., Manche, H., Laskar, J., \& Gastineau, M. 2008, A\&A, 477, 315

Fried, D. L. 1965, J. Opt. Soc. Am., 55, 1427

Haberreiter, M., Schmutz, W., \& Kosovichev, A. G. 2008, ApJ, 675, L53

Hauchecorne, A., Meftah, M., Irbah, A., et al. 2014, ApJ, 783, 127

Hestroffer, D., \& Magnan, C. 1998, A\&A, 333, 338

Hough, P. V. C. 1962, Method and means for recognizing complex patterns, U.S

Patent 3, 069, 654 (Washington: United States Atomic Energy Comission)

Ikhlef, R., Corbard, T., Morand, F., et al. 2016, MNRAS, 458, 517

Irbah, A., Corbard, T., Assus, P., et al. 2010, Proc. SPIE, 7735, 77356F

Irbah, A., Borgnino, J., Djafer, D., Damé, L., \& Keckhut, P. 2016, A\&A, 591, A150

Kolmogorov, A. 1941, Akad Nauk SSSR Dokl., 30, 301

Lamy, P., Prado, J.-Y., Floyd, O., et al. 2015, Sol. Phys., 290, 2617

Meftah, M., Corbard, T., Irbah, A., et al. 2014a, A\&A, 569, A60

Meftah, M., Hauchecorne, A., Crepel, M., et al. 2014b, Sol. Phys., 289, 1

Meftah, M., Hochedez, J.-F., Irbah, A., et al. 2014c, Sol. Phys., 289, 1043

Neckel, H. 2005, Sol. Phys., 229, 13

Prša, A., Harmanec, P., Torres, G., et al. 2016, AJ, 152, 41

Roddier, F. 1981, in Progress in Optics (Amsterdam: North-Holland Publishing Co.), 19, 281-376

Rozelot, J. P., Kosovichev, A., \& Kilcik, A. 2015, ApJ, 812, 91

Seidelmann, P. K. 1992, Explanatory Supplement to the Astronomical Almanac. A revision to the Explanatory Supplement to the Astronomical Ephemeris and the American Ephemeris and Nautical Almanac (Mill Valley, CA: University Science Books), 780

Sofia, S., Girard, T. M., Sofia, U. J., et al. 2013, MNRAS, 436, 2151

Tatarski, V. I. 1961, Wave Propagation in Turbulent Medium (New York: McGraw-Hill)

Vaquero, J. M., Gallego, M. C., Ruiz-Lorenzo, J. J., et al. 2016, Sol. Phys., 291, 1599

Vernazza, J. E., Avrett, E. H., \& Loeser, R. 1981, ApJS, 45, 635 


\section{Appendix A: PICARD/SODISM image during the 2012 transit of Venus}

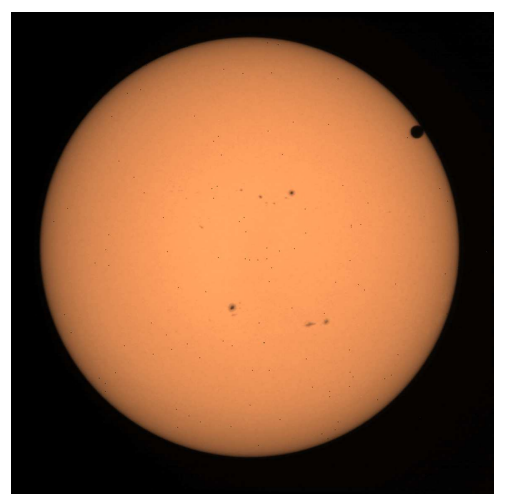

Fig. A.1. PICARD/SODISM image of the Sun (at $607.1 \mathrm{~nm}$ ) acquired on June 6, 2012, at 04H31 UTC during the Venus transit in 2012.

\section{Appendix B: Instrument and atmosphere system and convolution with the limb-darkening function}

\section{B.1. Optical transfer function of the instrument}

The optical transfer function $\left(H_{\mathrm{t}}\right)$ allows describing the formation of an image as a linear optical system in terms of angular frequency $(f)$. Equation (B.1) is applicable in the case of an optical system with a circular pupil $\left(D_{\mathrm{t}}\right)$ at a given wavelength $(\lambda)$ and for an incoherent illumination. $f_{c_{\mathrm{d}}}$ represents the diffraction cutoff frequency. Knowledge of the SODISM II PSF is fundamental.

$H_{\mathrm{t}}(f)=\frac{2}{\pi}\left[\arccos \left(\frac{f}{f_{\mathrm{c}_{\mathrm{d}}}}\right)-\frac{f}{f_{\mathrm{c}_{\mathrm{d}}}} \sqrt{1-\left(\frac{f}{f_{\mathrm{c}_{\mathrm{d}}}}\right)^{2}}\right]$

$f_{\mathrm{c}_{\mathrm{d}}}=\frac{D_{\mathrm{t}}}{\lambda}$

\section{B.2. Structure function of the wavefront phase perturbations}

A standard model of atmospheric turbulence used for observations was developed by Tatarski (1961) and by Fried (1965). This model is based on the work of Kolmogorov (1941) and is supported by a variety of experimental measurements. In the case of the Von Kármán model, the phase structure function $\left(D_{\emptyset}(\rho)\right)$ is given by Eq. (B.2), where $\rho$ represents an angular frequency (radians $^{-1}$ ) and $\mathcal{L}_{0}$ is the spatial coherence outer scale. This model depends on two optical atmospheric parameters $\left(r_{0}\right.$ and $\left.\mathcal{L}_{0}\right)$. When considering the propagation of an electromagnetic wave through a turbulent atmosphere verifying the Kolmogorov model, the structure function of the wavefront phase perturbations measured at ground level is given by Eq. (B.3) $\left(\mathcal{L}_{0} \rightarrow \infty\right)$; it is obtained for high values of the outer scale and depends only on the Fried parameter $\left(r_{0}\right)$,

$$
\begin{aligned}
D_{\emptyset}(\rho)= & {\left[1-1.485\left(\frac{\rho}{\mathcal{L}_{0}}\right)^{\frac{1}{3}}+5.383\left(\frac{\rho}{\mathcal{L}_{0}}\right)^{2}-6.281\left(\frac{\rho}{\mathcal{L}_{0}}\right)^{\frac{7}{3}}\right] } \\
& \times 6.88\left(\frac{\rho}{r_{0}}\right)^{\frac{5}{3}} \\
D_{\emptyset}(\rho)_{\mathcal{L}_{0} \rightarrow \infty}= & 6.88\left(\frac{\rho}{r_{0}}\right)^{\frac{5}{3}}
\end{aligned}
$$

\section{B.3. Total transfer function (instrument and atmosphere)}

The total transfer function $\left(\hat{F}_{\mathrm{T}} \rightarrow\right.$ instrument + atmosphere $)$ depends on the instrument optical transfer function $\left(H_{t}(f)\right)$ and the structure function based on the Kolmogorov model (Eq. (B.4)). $f_{\mathrm{c}_{\mathrm{t}}}$ represents the cutoff frequency associated with the turbulence.

$\hat{F}_{\mathrm{T}}(f)=H_{t}(f) \times \exp \left(-0.5 D_{\emptyset}(\lambda f)\right)$
$\hat{F}_{\mathrm{T}}(f)=H_{t}(f) \times \exp \left(-3.44\left(\frac{f}{f_{\mathrm{c}_{\mathrm{t}}}}\right)^{\frac{5}{3}}\right)$

$f_{\mathrm{c}_{\mathrm{t}}}=\frac{r_{0}}{\lambda}$

Impulse response function of the instrument-atmosphere system and convolution

The inverse Fourier transform of the total transfer function $\left(\hat{F}_{\mathrm{T}}\right)$ represents the spatial impulse response function of the instrument-atmosphere system. The solar limb $\left(\mathrm{LDF}_{\mathrm{t}}\right)$ is obtained by deriving the $2 \mathrm{D}$ convolution product of the solar limb shape $\left(\mathrm{LDF}_{\odot}\right)$ with the impulse response of the instrumentatmosphere system (Eq. (B.6)). The resulting solar radius will always be smaller than the "true value" of the Sun $\left(R_{\odot}\right)$,

$\mathrm{LDF}_{\mathrm{t}}=\mathrm{LDF}_{\odot} \otimes \mathcal{F}^{-1}\left[\hat{F}_{\mathrm{T}}\right]$

For PICARD/SODISM II, the Kolmogorov model is applicable if the spatial coherence outer scale is greater than $100 \times D_{\mathrm{T}}\left(\mathcal{L}_{0} \geqslant 10 \mathrm{~m}\right.$, thus $\left.\mathcal{L}_{0} \rightarrow \infty\right)$. Moreover, $r_{0}$ is related to the FWHM of the seeing disk. For a telescope whose pupil diameter $\left(D_{\mathrm{t}}\right)$ is much greater than $r_{0}$, we have $F W H M \simeq 0.98 \lambda / r_{0}$. When $r_{0} \rightarrow \infty$, the resolution is limited by diffraction $\left(F W H M \simeq 1.03 \lambda / D_{\mathrm{t}}\right.$ for a circular pupil and with Airy function). When $r_{0} \rightarrow 1 \mathrm{~cm}$ (poor observing site), it is understood that turbulence is a dominant effect and can have a catastrophic effect on the resolution of an image of a telescope. 